\title{
THE ORIENTATION OF THE DYNAMICAL REFERENCE FRAME
}

\author{
J. G. Williams, J. O. Dickey, X X Newhall, and E. M. Standish \\ Jet Propulsion Laboratory \\ California Institute of Technology \\ Pasadena, CA 91109-8099 USA
}

\begin{abstract}
We summarize the current status of the JPL ephemerides, focusing on the various data types utilized, especially the impact of the modern ranging data, and the resulting accuracies obtained. The dynamical equinox, as determined from the analysis of Lunar Laser Ranging data, is determined with an accuracy of 5 mas and the obliquity to a 2 mas level in $\sim 1983$, the weighted center of data. Knowledge of the lunar and planetary positions with respect to the dynamical equinox degrades to 10 mas at J2000. Twenty years of LLR data allow for the separation of the $18.6 \mathrm{yr}$ nutation terms from the precession constant. The correction to IAU precession is found to be $-2.7 \pm 0.4 \mathrm{mas} / \mathrm{yr}$, while the $18.6 \mathrm{yr}$ nutation of the pole is $3.0 \pm 1.5 \mathrm{mas}$ larger in magnitude than the 1980 IAU series. The necessity of different reference systems and the accurate knowledge of the interconnections between frames is addressed.
\end{abstract}

\section{Introduction}

This paper highlights recent JPL activities in the area of lunar/planetary ephemerides and related investigations. Section 2 discusses current JPL ephemerides with emphasis on the data sets considered, the orientation of the ephemerides, and ephemeris uncertainties; for a fuller discussion, the reader is referred to Williams and Standish (1989). The corrections to the IAU nutation and precession constants are presented in Section 3; a more detailed account is given by Williams et al. (1990). Reference frame issues are addressed in Section 4 (see Dickey, 1989 for a complete discussion); concluding remarks and a summary are given in Section 5.

\section{Ephemeris Considerations}

\subsection{OBSERVATIONAL DATA}

The creation of modem ephemerides is quite a different process from a few decades ago. We can now safely assume that our equations of motion properly describe the physical laws of gravitation and that all of the significant forces which affect the motions of the planets and Moon are known. Furthermore, we are no longer overwhelmed with the process of integrating the equations of motion. Now modern computers do the integration numerically without concem about problems such as non-converging expansions, neglected terms, and truncated series. Numerical integration programs have been tested; they provide sufficient accuracy.

Full concentration is now given to the observational data: the accuracy, variety, coverage and the reduction processes - those are the most important ingredients in creating modern-day ephemerides.

Table 1 lists the observational data now being fit to generate ephemerides. The table shows many additions since the creation of JPL's DE200/LE200 in 1980 as described by Standish (1990). These further sets of data are in the form of additions to standard data types as well as completely new types of observations. The acquisition and utilization of observational data continues to be the most vital part of the ephemeris creation process. 
As a result of the increasing strength of the observational data, JPL is presently engaged in the creation of its next major set of planetary and lunar ephemerides, expected to be available in late 1990. Significant improvements over DE200 are expected in the ephemerides of each planet as well as the Moon.

TABLE 1. THE SOURCES OF THE OBSERVATIONAL DATA

Sun Mer Ven Mar Jup Sat Ura Nep Plu Moon

Optical transits

Photoelectric transits

Astrolabe

Radar ranging

Mariner 9 Ranges

Mariner 10 Ranges

Viking Lander Ranges

Radio Astrometry

Ring Occultations

Disk Occultations

Pioneer Tracking Data

Voyager Tracking Data

Pluto Astrometry

Lunar Laser Ranging

$1911-$
$1982-$
$1969-$
$1964-$
$1971-72$
$1974-75$
$1976-82$
$1983-$
$1977-$
$1968-$
$1973-80$
$1979-89$
$1914-$
$1969-$

$\begin{array}{llllllll}\mathbf{S} & \mathbf{V} & \mathbf{M} & \mathbf{J} & \mathbf{S} & \mathbf{U} & \mathbf{N} & \\ & & \mathbf{M} & \mathbf{J} & \mathbf{S} & \mathbf{U} & \mathbf{N} & \mathbf{P} \\ \mathbf{M} & \mathbf{V} & \mathbf{M} & \mathbf{J} & \mathbf{S} & \mathbf{U} & & \\ \mathbf{M} & & \mathbf{M} & & & & & \\ & & \mathbf{M} & & & & & \\ & & & \mathbf{J} & \mathbf{S} & \mathbf{U} & \mathbf{N} & \\ & & & & & \mathbf{U} & \mathbf{N} & \\ & & & \mathbf{J} & \mathbf{S} & & & \\ & & & \mathbf{J} & \mathbf{S} & \mathbf{U} & \mathbf{N} & \\ & & & & & & & \mathbf{P}\end{array}$

$\mathbf{P}$

\subsection{ORIENTATION OF THE EPHEMERIDES}

Two important features of the ephemerides deserve mention, especially since these are neither well known nor intuitively apparent.

Inertial mean motions: The mean motions of the four inner planets and the Moon are determined from the ranging observations-for example, lunar laser, radar, and spacecraft-not by the optical observations; there are a number of explanations designed to show why (see, e.g., Standish and Williams, 1990). The most accurate planetary ranges are from Earth to Mars and the most accurate inertial mean motions are for these two planets (0:01/cty as given in Table 2).

Dynamical equinox and obliquity: The lunar laser ranging observations are highly sensitive to the direction of the pole of the Earth's rotation and, therefore, to the celestial equator of date. Data over an extended period of time will provide determinations of the Earth's orientation. The observations are also highly sensitive to the solar perturbations on the lunar orbit, which effectively cause the lunar orbit to precess along the ecliptic. Thus, the location of the ecliptic of date is also determined from the lunar observations. As a result, for successful data reduction, the true equator must be represented accurately in the analysis program and the true ecliptic in the ephemerides, most importantly over the time span of the lunar laser ranging data. Finally, one may locate the mean ecliptic at an epoch by analyzing the true ecliptic, given by the ephemeris of the Earth-Moon barycenter orbit about the Sun. This determination has been done by Standish (1982) and by Chapront-Touze and Chapront (1983) in order to extract the obliquity and dynamical equinox. The accuracy of the obliquity determination is better than 2 mas and, in the early $1980 \mathrm{~s}$, the Moon and planets are known with respect to the dynamical equinox to 5 mas. Recent obliquity determinations are close to the value in Standish (1982).

Thus, the ephemerides of the inner four planets and the Moon are created without reference to objects outside the solar system. The mean motions with respect to inertial space and the 
orientation to the mean equator and the dynamical equinox are determined strictly from ranging measurements coupled with dynamics.

\subsection{EPHEMERIS UNCERTAINTIES}

An analysis of the ephemeris parameter uncertainties by Williams and Standish (1989) has been made by considering the accuracies of the relevant observational data and by considering how sensitive such observations are to changes in each of the parameters. Those results are used here in producing Table 2, where the estimates are intended to be realistic uncertainties. We also include our estimates of the orbits of the outer planets, realizing that the uncertainty of extrapolating into the future is largest for the outermost planets.

TABLE 2. ESTIMATED EPHEMERIS ERRORS

\begin{tabular}{|c|c|c|c|c|c|}
\hline & Moon & Merc \& Ven & Mars & Jup...Nep & Pluto \\
\hline \multicolumn{6}{|l|}{ longitude } \\
\hline $\begin{array}{l}\text { wrt earth in } 1980 \\
\text { wrt earth in } 1990\end{array}$ & $\begin{array}{l}{[0: 001]} \\
{[0: " 001]}\end{array}$ & $\begin{array}{l}0: 002 \\
0.02\end{array}$ & $\begin{array}{l}0: 00002 \\
0: 001\end{array}$ & $\begin{array}{l}0.05 \\
0.05\end{array}$ & $\begin{array}{l}0.5 \\
0.5\end{array}$ \\
\hline $\begin{array}{l}\text { wrt } 1980 \text { dyn eq } \\
\text { wrt } 2000 \text { dyn eq }\end{array}$ & $\begin{array}{l}0.005 \\
0.01\end{array}$ & $\begin{array}{l}0 . " 05 \\
0.01\end{array}$ & $\begin{array}{l}0.005 \\
0.01\end{array}$ & $\begin{array}{l}0.05 \\
0.05\end{array}$ & $\begin{array}{l}0.5 \\
0.5\end{array}$ \\
\hline latitude & 0.002 & 0."02 & 0."0005 & 0.05 & 0.5 \\
\hline mean motion & $\begin{array}{l}0.04 / \text { cty } \\
1 . / \text { cty }^{2}\end{array}$ & $0.2 /$ cty & 0.01/cty & $0.5 /$ cty & $2 " / c t y$ \\
\hline
\end{tabular}

\section{Precession and Nutation}

Twenty years (August 1969 to December 1989) of lunar laser ranges have been analyzed to extract corrections to the luni-solar precession constant and the $18.6 \mathrm{yr}$ nutation coefficients. The twodecade span of data permits the precession and $18.6 \mathrm{yr}$ nutations to be separated; the largest correlation between them being -0.64 . The most recent ranges are an order of magnitude more accurate than the ranges from the early 1970 s, and the data are weighted accordingly.

The solutions given in Table 3 are from Williams et al. (1990), where additional details may be found. The nominal precession and nutation expressions are given by the respective IAU working groups (Lieske et al., 1977, Seidelmann, 1982). The table gives the corrections to these standard values.

Three solutions are presented. Case $A$ is a solution for the precession, in-phase and out-ofphase $18.6 \mathrm{yr}$ nutation coefficients, and the two in-phase annual components. Case B is based on the recent improvements to the nutation theory by Kinoshita and Souchay (1990). Their corrections to the $9.3 \mathrm{yr}$ coefficients and the in-phase $18.6 \mathrm{yr}$ obliquity coefficient were adopted; the out-ofphase $18.6 \mathrm{yr}$ coefficient corrections were constrained to a fixed ratio, additional in-phase $18.6 \mathrm{yr}$ coefficient corrections were constrained to a ratio which depends on the value of the precession correction; and the annual corrections were fixed at values similar to those found by VLBI. Case C is based on the recent improvements to the nutation theory by Zhu et al. (1990). Their corrections were adopted for the $18.6 \mathrm{yr}$ (in-phase obliquity), $9.3 \mathrm{yr}$, annual (in-phase), semi-annual, and 
semi-monthly coefficients. Analogous to Case B, two constraints were set up for ratios of the 18.6 yr coefficients.

The three solutions of Table 3 show that the precession constant needs to be decreased and the magnitude of the 18.6 yr nutation needs to be increased. The out-of-phase corrections of the 18.6 yr terms are similar to the size expected from ocean tide influences (Wahr and Sasao, 1981, Zhu et al., 1990), but the errors only permit us to say that it is suggestive. Case A indicates that the annual correction is detected. An additional solution with the two annual components constrained to be equal gives $1.8 \pm 0.5$ mas. If the recent theoretical improvements to nutations are an accurate representation of the motion of the Earth's axis of rotation, then the solutions of Cases B and C are expected to be improvements over the Case A solution. The uncertainties given in the table are intended to be realistic estimates; the formal errors are smaller.

The four LLR-derived corrections to the $18.6 \mathrm{yr}$ coefficients are in good agreement with the recent VLBI results of Herring et al. (1990), and their precession correction of $-3.2 \pm 1.3 \mathrm{mas} / \mathrm{yr}$ is compatible with our value. This volume (Charlot et al.) has a combined LLR and VLBI solution which adds the strength of the LLR data for precession to the accuracy of the VLBI data for short periods.

The $-2.7 \mathrm{mas} / \mathrm{yr}$ correction to the luni-solar precession constant gives a new value of $50.3851 " / y r$ at $\mathrm{J} 2000$. It is the luni-solar precession rather than the general precession which is determined. The dynamical equinox is better determined during the past decade than at $\mathrm{J} 2000$. The uncertainty of the precession constant contributes to the 10 mas uncertainty at J2000 in Table 2.

TABLE 3. CORRECTIONS TO PRECESSION AND NUTATIONS

\begin{tabular}{lccc}
\hline & Case A & Case B & Case C \\
\hline Precession (mas/yr) & $-2.8 \pm 0.5$ & $-2.7 \pm 0.4$ & $-2.7 \pm 0.4$ \\
In-phase 18.6 yr terms (mas) & & & \\
$\quad \Delta \varepsilon$ & $0.4 \pm 1.9$ & $3.1 \pm 1.1$ & $3.0 \pm 1.2$ \\
$\quad \sin \varepsilon \Delta \psi$ & $-3.4 \pm 1.6$ & $-3.4 \pm 1.5$ & $-3.1 \pm 1.5$ \\
Out-of-phase 18.6 yr terms (mas) & & \\
$\quad \Delta \varepsilon$ & $1.2 \pm 1.9$ & $1.4 \pm 1.6$ & $1.3 \pm 1.6$ \\
$\quad \sin \varepsilon \Delta \psi$ & $1.5 \pm 1.9$ & $1.0 \pm 1.2$ & $1.0 \pm 1.3$ \\
In-phase annual terms (mas) & & & $1.79 *$ \\
$\Delta \varepsilon$ & $1.6 \pm 1.1$ & $1.8^{*}$ & $1.89^{*}$ \\
$\sin \varepsilon \Delta \psi$ & $2.0 \pm 0.8$ & $1.8^{*}$ & \\
\hline
\end{tabular}

*Correction fixed at this value

\section{Reference Frame Considerations}

Each technique observing a particular class of objects can be expected to establish its own reference frame (see Table 4). Contemporary astronomy has led to the development of three types of celestial coordinate systems: the optical frame (e.g. FK4/FK5) based on the positions of galactic stars, 
TABLE 4. FRAME DETERMINATIONS

\begin{tabular}{lll}
\hline FRAME & TECHNIQUE & TARGET \\
\hline * Ephemeris & * Planetary/Spacecraft Ranging & * Planets \\
& * Lunar Laser Ranging & * Moon \\
* Optical & * Optical Astrometry & * Stars, Sun and Planets \\
* Radio & * Very-Long-Baseline & * Quasars, Radio Stars, \\
& Interferometry & $\begin{array}{c}\text { Pulsars, and Inter- } \\
\text { planetary Spacecraft }\end{array}$ \\
* Satellite & * Satellite Laser Ranging & * Earth-Oriting Satellite \\
& * Goppler & * Transmitting Satellites \\
\hline
\end{tabular}

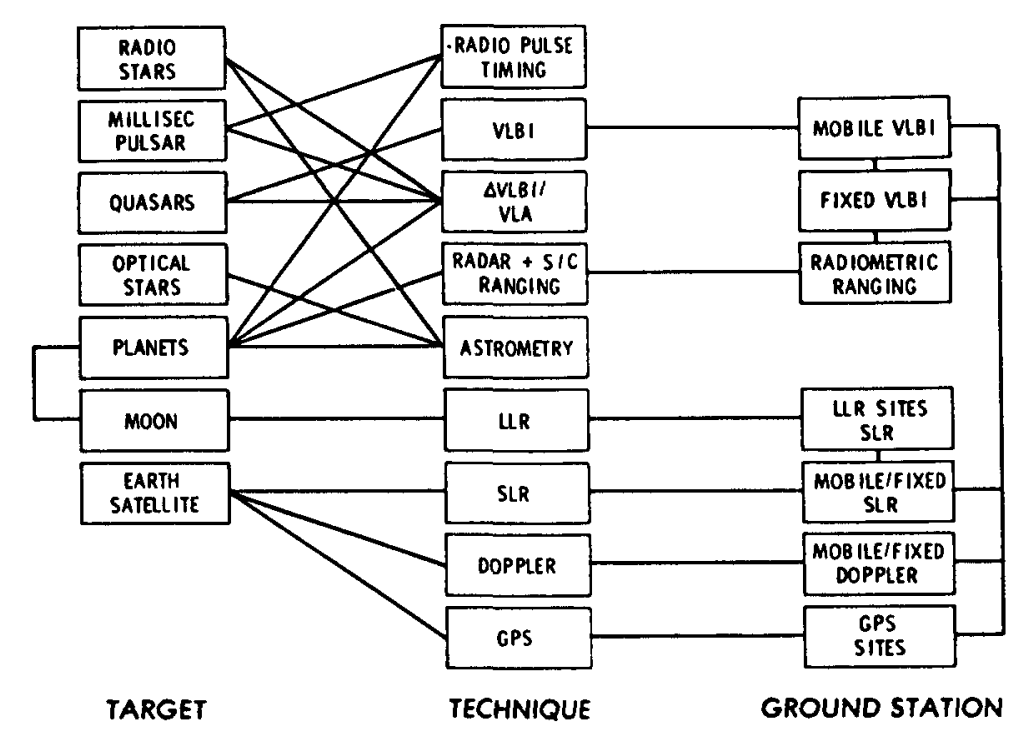

FIG. 1: Connections Between Reference Systems 
planets, and the Sun; the planetary/unar ephemeris frame based on the major celestial bodies of the solar system; and the radio frame constructed from observations of extragalactic sources/quasars. It should be noted that the radio, optical, and ephemeris frames generate complementary terrestrial frames as well. Other terrestrial frames are developed through the analysis of the data from Earthorbiting satellites [e.g. GPS (Global Positioning System), Doppler, and laser-reflecting satellites such as LAGEOS]. The terrestrial frames must consider local deformations as well as tectonic motion; for example, most of the sites are moving at rates of several $\mathrm{cm} / \mathrm{year}$. The celestial and terrestrial coordinate systems from a single technique and class of target are related through adopted constants and definitions. Each frame is rotated with respect to the others, and this offset may be time variable (e.g. the radio vs the FK4 frame).

Measurements are inherently more accurate in their "natural" frame and hence should always be reported as such. However, to benefit from the complementarity of the various techniques, knowledge of the frame interconnections (both the rotation and the time-variable offset) is essential; these are summarized in Fig. 1 and Table 4 (after Dickey, 1989). Recent activity in this area is indicated by the number of boxes and lines in Fig. 1 (the accuracy cut-off here is $0.05 \mathrm{arcsec}$ ). The lunar/planetary system, integrated in a joint ephemeris, is by its nature unified by the dynamics. The radio frame is tied to the ephemeris frame in several ways: one is via differential VLBI measurements of planet-orbiting spacecraft and angularly nearby quasars; another is the determination of a pulsar's position in the ephemeris frame (via timing measurements) and the radio frame [via radio interferometry (VLA)]. VLA observations of the outer planets (Jupiter, Satum, Uranus and Neptune) or their satellites provide an additional tie between these two frames. As for an optical-radio frame tie, a preliminary link has been established between the FK5 optical frame and the JPL radio reference frame via the differential VLBI measurement of optically bright radio stars and angularly nearby quasars coupled with comparisons of their optical positions, and also by the use of the optical positions of quasars. The optical and ephemeris frames are tied by optical observations of the planets. For a fuller discussion of these topics and referrals to references in the literature, the reader should see Dickey (1989).

\section{Concluding Remarks}

A summary of the current ephemeris developments at JPL was presented, stressing data types utilized and the accuracies obtained. Ranging observations are the dominant data for the inner four planets and the Moon; the most accurate ranges (and orbits) are for Earth, Mars, and the Moon. Optical data are significant for only the five outermost planets. The inclusion of Voyager tracking data and radio astrometry provide major improvements in the outer planet ephemerides.

Lunar laser ranging, being sensitive to the planes of the ecliptic, the lunar orbit, and the equator determines their mutual orientation to an accuracy of 2 mas and hence, the dynamical equinox (the intersection of the mean equator and the mean ecliptic) to an accuracy of 5 mas early in the 1980s. The uncertainty of the Moon and planets with respect to the dynamical equinox degrades to 10 mas at J2000. The dynamical equinox is used as our reference point for origin of the right ascension. The analysis of two decades of lunar laser ranging data has permitted the separation of the precession constant from the 18.6 year nutation terms. The correction to the IAU-adopted precession constant is $-2.7 \pm 0.4$ milliarcseconds/yr, whereas the $18.6 \mathrm{yr}$ nutation of the pole is found to be $3.0 \pm 1.5$ mas larger in magnitude than the 1980 IAU series. The nutation series corrections are consistent with theoretical expectations.

Reference frame issues were addressed briefly. It was stressed that each technique acquires measurements in its own reference frame, with results being most accurate in its own "natural" frame. To benefit from the complementarity of the various techniques, knowledge of the frame interconnections is essential.

ACKNOWLEDGEMENTS. The work described in this paper was performed at the Jet Propulsion Laboratory, California Institute of Technology, under contract with the National Aeronautics and Space Administration. 


\section{References}

Chapront-Touzé, M. and Chapront, J., The lunar ephemeris ELP2000, Astron. Astrophys., 124, $50-62,1983$.

Charlot, P., Sovers, O.J., Williams, J.G., and Newhall, X X, A Global VLBI/LLR Analysis for the Determination of Precession and Nutation Constants, this volume.

Dickey, J.O., Intercomparisons Between Kinematic and Dynamical Systems, in Reference Frames (J. Kovelevsky, I.I. Mueller and B. Kolaczek, eds.) Kluwer Academic Publishers, Dordrecht, 305-326, 1989.

Herring, T.A., Buffett, B.A., Matthews, P.M., and Shapiro, I.I., J. Geophys. Res., in press, 1990.

Kinoshita, H. and Souchay, J., Celes. Mech., in press, 1990.

Lieske, J.H., Lederle, T., Fricke, W., and Morando, B., Astron. Astrophys., 58, 1, 1977.

Seidelmann, P.K., Celes. Mech., 27, 7, 1982.

Standish, E. M., The JPL Planetary Ephemerides, Cel. Mech. J., 26, 181-186, 1982 .

Standish, E.M., The observational basis for JPL's DE200, the planetary ephemerides of the Astronomical Almanac, Astron. Astrophys., 233, \#1, 252-271, 1990.

Standish, E.M. and Williams, J.G., Dynamical Reference Frame in the Planetary and Earth-Moon Systems, in Inertial Coordinate System on the Sky (J..H. Lieske and V.K. Abalakin, eds.) Kluwer Academic Publishers, Dordrecht, 173-181, 1990.

Standish, E.M., Orientation of the JPL Ephemerides, DE200/LE200, to the Dynamical Equinox of J2000, Astron. Astrophys., 114, 297-302, 1982.

Wahr, J.M. and Sasao, T., Geophys. J. Royal Astron. Soc., 64, 747, 1981.

Williams, J.G. and Standish, E.M., Dynamical Reference Frames in the Planetary and Earth-Moon Systems, in Reference Frames (J. Kovalevsky, I.I. Mueller and B. Kolaczek, eds.) Kluwer Academic Publishers, Dordrecht, 67-90, 1989.

Williams, J.G., Newhall, X X, Dickey, J.O., Luni-solar Precession: Determination from Lunar Laser Ranging, Astron. Astrophys. Lett., in press, 1990.

Zhu, S.Y., Groten, E., and Reigber, C., Astron. J., 99, 1024, 1990. 